\title{
Long non-coding RNA MEG3 suppresses survival, migration, and invasion of cervical cancer
}

This article was published in the following Dove Press journal:

OncoTargets and Therapy

\author{
Xiuhui Chen' \\ Junying $Q u^{2}$ \\ 'Department of Obstetrics and \\ Gynecology, Shuguang Hospital \\ Affiliated to Shanghai University \\ of Traditional Chinese Medicine, \\ Shanghai 20002I, China; '2Department \\ of Obstetrics and Gynecology, Ist \\ Affiliated Hospital of Fujian Medical \\ University, Fuzhou 350005, China
}

Correspondence: Xiuhui Chen Shanghai Shuguang Hospital Affiliated to Shanghai University of Traditional Chinese Medicine, Shanghai 20002I, China

Tel +86 2I 53821650

Email xiuhui_ch@I26.com
Background: Long non-coding RNAs nowadays emerge as important biomarkers or potential therapeutic targets discussed in human cancers. Among them, maternally expressed gene 3 (MEG3) is known to be decreased in a variety of malignancies, and this affects tumor cellular proliferation, migration, and invasion.

Materials and methods: Quantitative real-time PCR was performed to detect the expression of MEG3 in normal cervical epithelium, cervical intraepithelial neoplasia, and cervical squamous cell carcinoma tissues. Gain-of-function and loss-of-function studies were carried out to determine the effect of MEG3 on cell survival, migration, and invasion, which was evaluated by CCK-8 assay, wound healing assay, and transwell assays. mRNA and protein expression of Rac1 were finally determined by quantitative real-time PCR and immunoblotting, respectively. In addition, rescue experiments were performed by overexpression of Rac1.

Results: The expression of MEG3 was downregulated in cervical intraepithelial neoplasia and squamous cell carcinoma tissues. Forced expression of MEG3 led to reduced abilities of cell survival. Overexpression of MEG3 also inhibited cell migration and invasion in vitro. Cell proliferation marker and EMT markers were changed consistently with the phenotype. In addition, Rac1 was inhibited by MEG3 overexpression at both transcriptional and translational levels. Also, Rac1 could rescue the phenotype caused by long non-coding RNA MEG3. And, it negatively correlated with MEG3 expression in cervical cancer (CC) tissues and cell lines.

Conclusion: Our findings revealed that MEG3 could negatively regulate $\mathrm{CC}$ cell survival, migration, and invasion. It might serve as an important target for $\mathrm{CC}$ treatment.

Keywords: MEG3, cervical cancer, survival, migration and invasion, Rac1

\section{Introduction}

According to the statistics, cervical cancer (CC) originating from the female reproductive system is the third most common malignancy and fourth leading cause of cancer-related death in women all around the world. ${ }^{1,2}$ Every year there are over 500,000 cases diagnosed as CC and approximately 200,000 deaths caused by CC in the world, with the majority in developing countries. ${ }^{2}$ Long-term infection by human papillomavirus (HPV) is an important factor for the initiation of CC. However, existing CC screening toward HPV has not obviously brought down the diagnostic rate of this disease, indicating other factors also contribute to the development of CC. ${ }^{3-5} \mathrm{CC}$ could be cured by surgery, radiotherapy, and concurrent platinum-based chemotherapy. Nevertheless, some cases develop recurrence, mainly attributed to metastasis, and receive bad outcomes. ${ }^{6}$ Therefore, novel and efficient therapeutic targets are required for its treatment.

Recent research progress in the human genome has found non-coding RNAs (ncRNAs) transcribed from $<2 \%$ of the total genome with limited or no protein-coding capacity 
due to lack of open reading frames (ORFs). ${ }^{7,8}$ Long non-coding RNAs (lncRNAs) are a large subgroup of ncRNAs with more than 200nt in length, and not necessarily conserved among different species. The function of most lncRNAs remains obscure, and their relevance to human diseases is largely undefined. Increasing evidence has demonstrated that some lncRNAs play vital roles in basic cellular functions, including cell proliferation, survival, differentiation, motility and apoptosis, and serve as potential oncogenes or tumor suppressors in cancers. ${ }^{9-11}$ However, the regulation of most 1 ncRNAs and their exact mechanisms in $\mathrm{CC}$ are not clear. Exploration of the function and involvement of IncRNA in CC would improve its therapy and enable new targets to be identified.

Maternally expressed gene 3 (MEG3), located on human chromosome $14 \mathrm{q} 32$, is a maternally expressed imprinted gene expressed in many normal tissues. ${ }^{12}$ It has been reported that MEG3 functions as a tumor suppressor since it is disrupted in various human cancers by epigenetic regulation. ${ }^{13-15}$ In $\mathrm{CC}$, antitumoral activity has been mentioned in previous studies, like inhibiting cell proliferation and inducing apoptosis. ${ }^{16,17}$ However, whether it is involved in migration and invasion remains elusive. Rac family small GTPase 1 (Rac1) encodes a GTPase which belongs to the RAS superfamily of small GTP-binding proteins. Studies have established that Rac1 regulates a diverse array of cellular events in cancer, such as controlling cell growth, cytoskeletal reorganization, and metastasis. ${ }^{18,19}$ Herein, through a series of experiments, we verified that MEG3 is decreased in CC samples, and suppresses cell survival, migration, and invasion by targeting Rac1. Our findings strengthen the antitumoral role of MEG3 in CC.

\section{Materials and methods}

\section{Patients and tissues}

A total of 20 normal cervical epithelium (NCE) tissue samples, 20 cervical intraepithelial neoplasia (CIN) tissue samples, and 20 cervical squamous cell carcinoma (SCC) tissue samples were obtained from patients in the 1st Affiliated Hospital of Fujian Medical University from January 2015 to December 2016. The study was approved by the Medical Ethics Committee of the 1st Affiliated Hospital of Fujian Medical University. Written informed consent from all patients was obtained prior to participation in the study. All samples were immediately snap-frozen, and kept at $-80^{\circ} \mathrm{C}$ for quantitative real-time PCR (qRT-PCR) until use.

\section{Cell culture}

Non-tumoral human cervical epithelial (HcerEpic) cells and human cervical carcinoma cell lines C-33A, C4-1, Caski,
SiHa, and Hela were obtained from the American Type Culture Collection (ATCC, Manassas, VA, USA) or the Chinese Academy of Sciences Cell Bank (Shanghai, China). Cells were cultured in 1,640 or DMEM medium (HyClone, Victoria, Australia) supplemented with 10\% FBS (HyClone), 100 units $/ \mathrm{ml}$ of penicillin $\left(\mathrm{Gibco}^{\circledR}\right.$, Thermo Fisher Scientific, Waltham, MA, USA), and $100 \mathrm{mg} / \mathrm{mL}$ of streptomycin (Gibco, Thermo Fisher Scientific) at $37^{\circ} \mathrm{C}$, in a humidified atmosphere containing $5 \% \mathrm{CO}_{2}$ in an incubator.

\section{qRT-PCR}

Total RNAs were isolated from cells by Trizol reagent (Thermo Fisher Scientific), and reverse transcriptions were performed by GoScript ${ }^{\mathrm{TM}}$ Reverse Transcription System (Promega Corporation, Fitchburg, WI, USA) as per the manufacturer's instructions. qRT-PCR was performed using SYBR ${ }^{\circledR}$ Green Realtime PCR Master Mix (Toyobo, Osaka, Japan) on an Applied Biosystems 7,500 Real-Time PCR system (Thermo Fisher Scientific). The U6 small RNA or $\beta$-actin mRNA was used as internal controls of lncRNAs or genes. All reactions were run in triplicate. The $2^{-\Delta \Delta \mathrm{Ct}}$ method was used to calculate the relative expression of each reaction. Primers used for amplification are provided as follows:

\section{lncRNA MEG3}

Forward: 5'-GCCAAGCTTCTTGAAAGGCC-3' Reverse: 5'-TTCCACGGAGTAGAGCGAGTC-3'

U6 small RNA

Forward: 5'-CGCTTCGGCAGCACATATAC-3'

Reverse: 5'-T TCACGAATTTGCGTGTC AT-3'

Rac1

Forward: 5'-AGGAAGGCGGACATATTAGTCCCT-3' Reverse: 5'-AGACG ATAGTTGGGTC CCGGC-3'

$\beta$-actin

Forward: 5'-TGACGGGGTCACCCACACTGTGC-3' Reverse: 5'-CTAGAAGCATTTGCGGTGGACGA-3'

\section{Plasmids, siRNAs, and transfections}

Overexpression of lncRNA MEG3 plasmid and Rac1 plasmid were from GeneChem Company (Shanghai, China). Empty vector was used as a control. Two MEG3 siRNAs (siMEG3 \#1, and siMEG3 \#2) and scrambled negative control siRNA (NC) were purchased from Genepharma (Shanghai, China). Transfections were performed by using Lipofectamine 2000 
Transfection Reagent (Thermo Fisher Scientific) according to the manufacturer's instructions.

\section{Cell proliferation assay}

Cells were seeded at a density of $3 \times 10^{3}$ cells per well in a 96-well plate containing $100 \mu \mathrm{L} 1,640$ or DMEM full medium. Cell Counting Kit-8 (Dojindo, Kumamoto, Japan) reagents was added at 1 day, 2 days, 3 days, 4 days and 5 days after seeding ( 0 day) and incubated at $37^{\circ} \mathrm{C}$ for 2 hours. The data of OD 450 were measured by a microplate reader (Molecular Devices LLC, Sunnyvale, CA, USA). Each experiment was performed at least three times with six replicates.

\section{Wound healing assay}

Cervical cells were seeded into 6-well plates. Cells were scraped with a sterile $200 \mu \mathrm{L}$ micropipette tip when grown to $90 \%$ confluent. The wounded monolayers were washed with phosphate buffer solution (PBS) twice to remove cell debris. The distance between the two edges of the wound was calculated at five different positions. After 48 hours, the distance between the two edges was measured again at each position.

\section{Transwell assay}

Transwell chambers $\left(\right.$ Costar $^{\circledR}$, Corning Incorporated, Corning, NY, USA) with $8 \mu \mathrm{m}$ pore polycarbonate filters were coated with matrigel (BD Biosciences, San Jose, CA, USA). Into the upper chambers in serum-free medium, $3 \times 10^{4}$ cells were seeded at a volume of $200 \mu \mathrm{L} /$ well. The lower chamber contained $500 \mu \mathrm{L}$ medium with $10 \%$ FBS as a chemoattractant. Cells on the upper surface of the chamber were removed by a cotton swab after incubating for 48 hours at $37^{\circ} \mathrm{C}$ with $5 \% \mathrm{CO}_{2}$. Traversed cells on the lower side of the filter were fixed with $4 \%$ paraformaldehyde and stained with $0.25 \%$ crystal violet solution. Invaded cells in the lower chamber were counted and photographed using phase contrast microscopy. The numbers of invaded cells were determined by photographing in five random views per chamber at $200 \times$ magnification.

\section{Immunoblotting}

Cells were collected and resuspended in RIPA lysis buffer (Beyotime, Beijing, China). A total of $30 \mu \mathrm{g}$ of total protein was loaded and separated on SDS-polyacrylamide gels (SDS-PAGE) and transferred onto polyvinylidene difluoride membranes (Millipore, Billerica, MA, USA). The membranes were blocked with $5 \%$ non-fat milk for 1 hour at room temperature and incubated with specific primary antibodies at $4{ }^{\circ} \mathrm{C}$ overnight. The specific antibodies for Rac1, PCNA, E-cadherin, $\mathrm{N}$-cadherin, vimentin, and $\beta$-actin proteins were from Cell Signaling Technology (Cell Signaling Technology Inc., Danvers, MA, USA). The membranes were incubated with the specific HRP-conjugated secondary antibodies (Beyotime) for 1 hour at room temperature. ECL chromogenic substrate (Beyotime) was used to visualize the bands. $\alpha$-tubulin was used as an internal control.

\section{Statistical analysis}

Statistical analyses were performed using SAS v9.2. All graphs were made using GraphPad software v6.0. All data from three independent experiments were expressed as mean \pm SD. Differences between groups were performed by two-tailed Student's $t$-test. The relationship between lncRNA MEG3 and Rac1 expression was performed by linear regression. A $P$-value of $<0.05$ was considered statistically significant.

\section{Results}

\section{IncRNA MEG3 is downregulated in CC tissues and cell lines}

Firstly, to understand the function of lncRNA MEG3 in CC, we detected the expression of MEG3 in 20 cases of NCE, 20 cases of CIN, and 20 cases of cervical SCC obtained from individuals with informed content. The qRT-PCR result showed that MEG3 expression was significantly downregulated in CINs compared with NCEs and further declined in SCCs, suggesting a disease progression-dependent decrease (Figure 1A). On the other hand, qRT-PCR detection in five CC cell lines (C-33A, C4-1, Caski, SiHa, and Hela) also confirmed its downregulation, compared with non-tumoral human cervical epithelial HcerEpic cells (Figure 1B). These findings indicated that MEG3 was decreased in CC and suggested its antitumoral effects.

\section{IncRNA MEG3 inhibits CC cell survival}

Based on the above results, we questioned whether lncRNA MEG3 could impact on CC cell survival. We mainly utilized CCK-8 assay to evaluate cell survival, namely, cell viability. As shown in Figure 2A, we obtained successfully MEG3 overexpressing subcell lines in Hela and SiHa. Meanwhile, we found that MEG3 overexpression led to an obviously lower cell survival rate in both cell lines (Figure 2B). Moreover, the proliferation marker PCNA showed significant decrease after MEG3 overexpression 

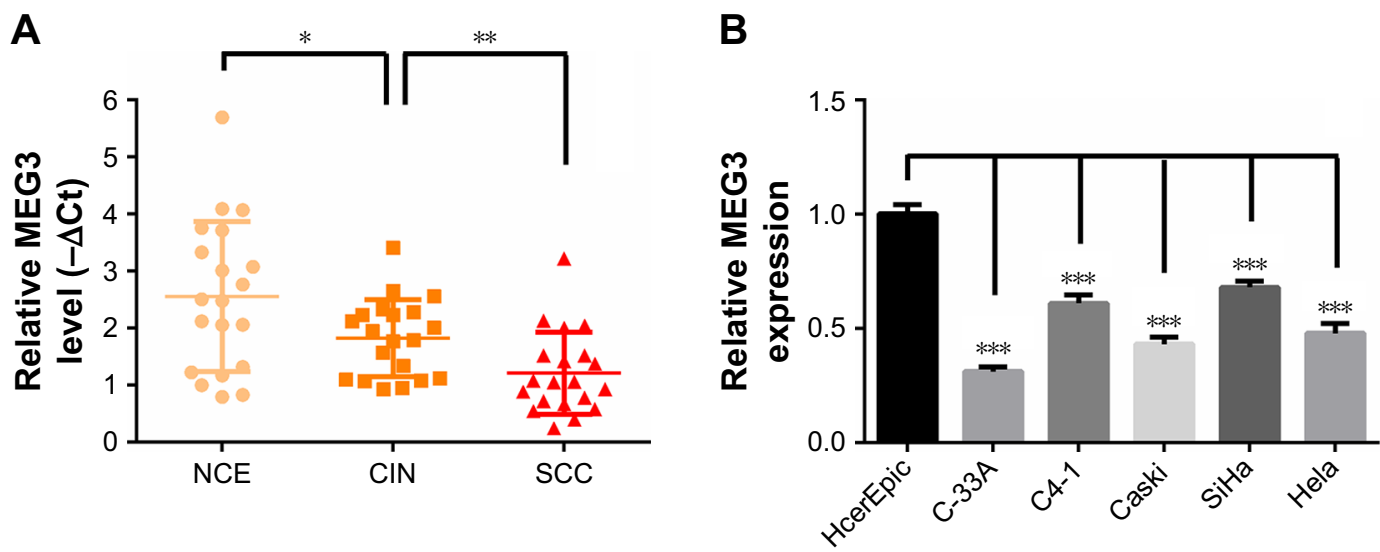

Figure I IncRNA MEG3 is downregulated in CC tissues and cell lines.

Notes: (A) qRT-PCR analysis of expression of IncRNA MEG3 expression in NCE, CIN and SCC tissue samples ( $\mathrm{n}=20$ ). (B) qRT-PCR analysis of expression of IncRNA MEG3 expression in non-tumoral HcerEpic and human cervical carcinoma cells (C-33A, C4-I, Caski, SiHa, and Hela). $* P<0.05, * * P<0.0$ I, $* * * P<0.00 \mathrm{I}$.

Abbreviations: NCE, normal cervical epithelium; CIN, cervical intraepithelial neoplasia; SCC, squamous cell carcinoma; CC, cervical cancer; HcerEpic, human cervical epithelial cells; IncRNAs, long non-coding RNAs; qRT-PCR, quantitative RT-PCR; MEG3, maternally expressed gene 3.

in both cells (Figure 2C). This finding is in line with our forecast, because its antitumoral activity has been mentioned in previous studies in CC, including inhibiting cell proliferation and inducing apoptosis. ${ }^{16,17}$

\section{IncRNA MEG3 inhibits migration and invasion of CC cells}

Considering migration and invasion are vital steps guiding cancer cell metastasis, we then sought to determine whether
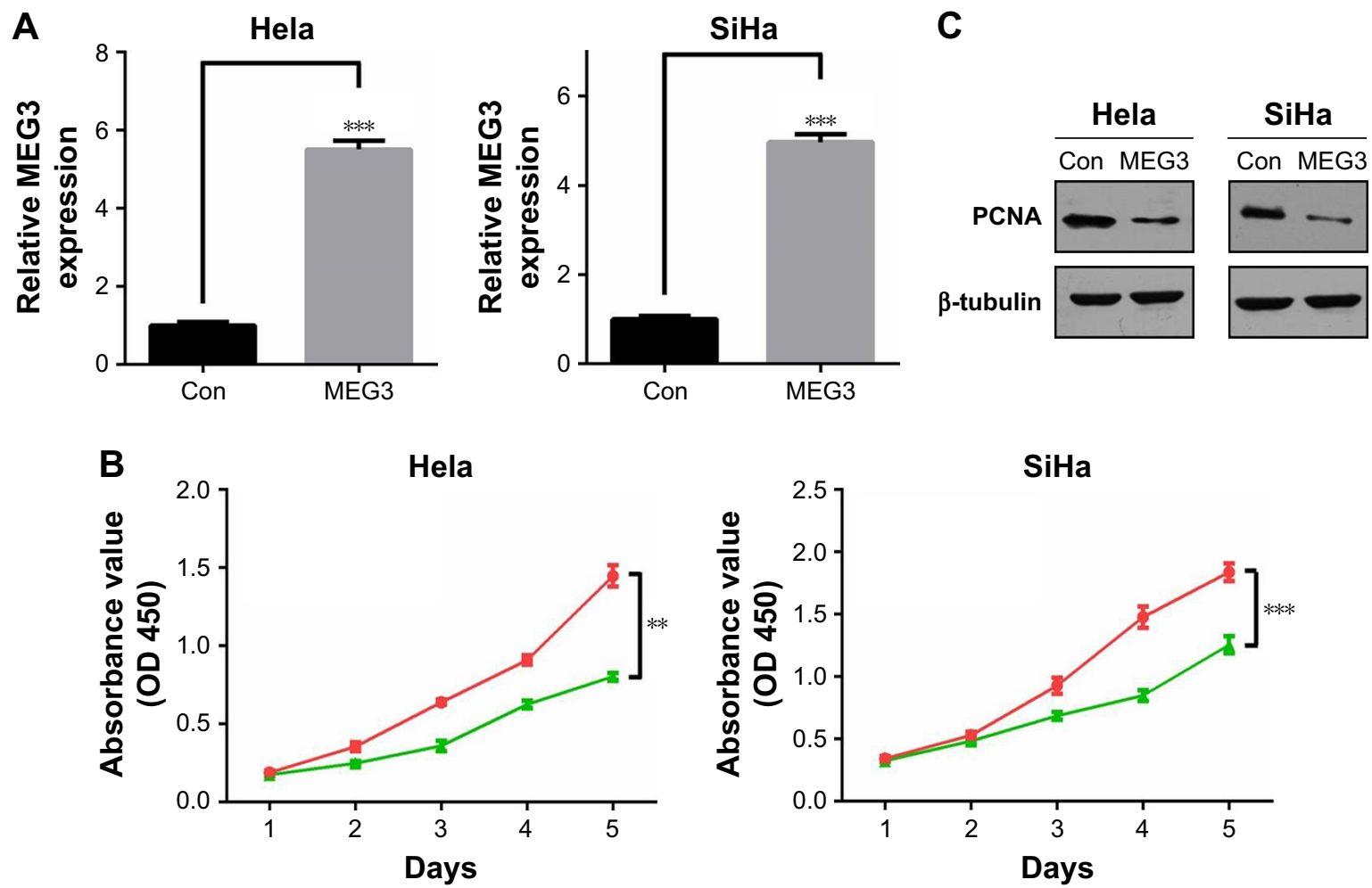

Con - MEG3

Figure 2 IncRNA MEG3 inhibits cell survival of CC cells.

Notes: (A) Expression of IncRNA MEG3 level was detected by qRT-PCR in Hela and SiHa cells transfected with MEG3 overexpression plasmid and control plasmid (con). (B) CCK-8 assay was performed in Hela and SiHa cells transfected with MEG3 overexpression plasmid and control plasmid (con). (C) Immunoblotting assay was performed in Hela and SiHa cells transfected with MEG3 overexpression plasmid and control plasmid (con). $* * P<0.01, * * * P<0.001$.

Abbreviations: Con, control; IncRNA, long non-coding RNA; qRT-PCR, quantitative real-time PCR; MEG3, maternally expressed gene 3; CC, cervical cancer; PCNA, proliferating cell nuclear antigen. 
A

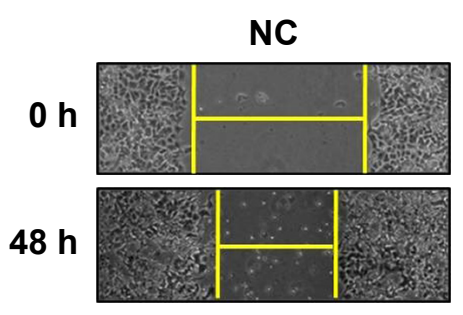

B
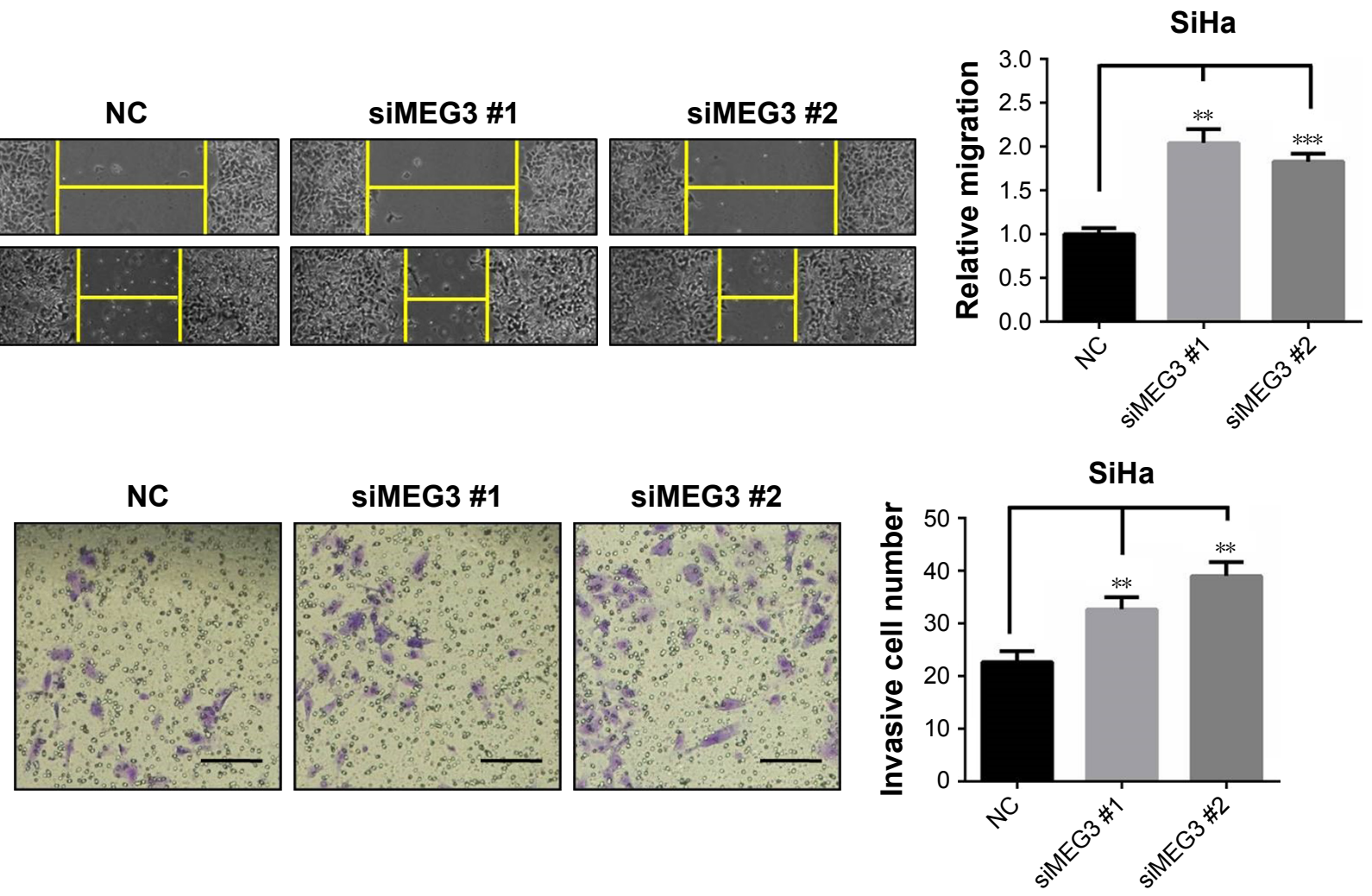
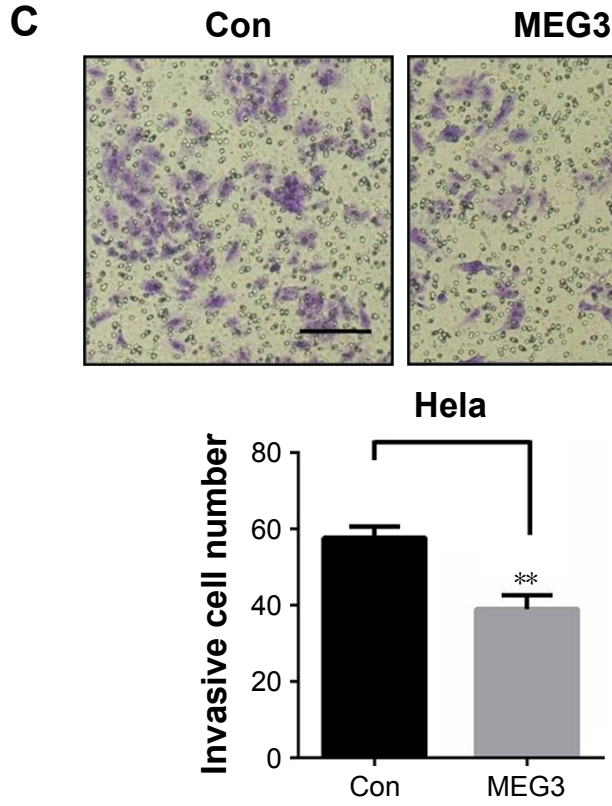

MEG3
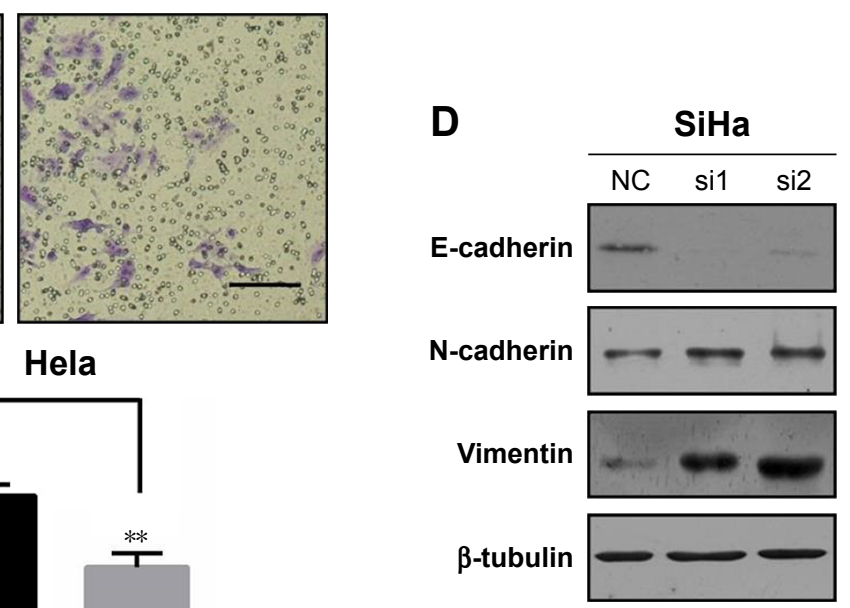

Hela

Con MEG3
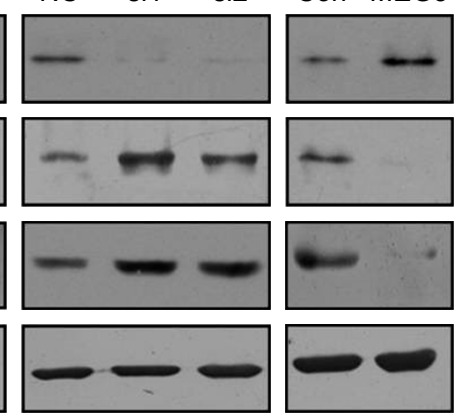

Figure 3 IncRNA MEG3 inhibits migration and invasion of CC cells.

Notes: (A) Knockdown of IncRNA MEG3 improved the cell migration in SiHa. (B) Knockdown of IncRNA MEG3 improved the cell invasion in SiHa. (C) Overexpression of IncRNA MEG3 inhibited the cell invasion in Hela. (D) Immunoblotting assay was performed in Hela or SiHa cells transfected with MEG3 siRNAs or overexpression plasmid. Data are presented as mean $\pm \mathrm{SD}$. $* * P<0.01$, $* * * P<0.001$. Scale bar: $50 \mu \mathrm{m}$.

Abbreviations: IncRNAs, long non-coding RNAs; MEG3, maternally expressed gene 3; Con, control; NC, negative control.

lncRNA MEG3 also impacted on these two processes of CC cells in vitro. We knocked down the intracellular MEG3 by two independent siRNAs. As shown in Figure 3A and B, loss of MEG3 significantly improved migration assessed by wound healing assay and invasion by transwell assays in $\mathrm{SiHa}$ cells. Besides, MEG3 overexpression in Hela cells inhibited invasion in vitro (Figure 3C). Immunoblotting assay was performed to detect the EMT markers changing. The results showed that E-cadherin was decreased while N-cadherin and vimentin increased significantly after knocking down 
of MEG3. Also, overexpressing of MEG3 did the opposite (Figure 3D). This finding was the first time in CC. Our results put forward another important role of lncRNA MEG3 in suppressing cell metastasis of CC cells.

\section{IncRNA MEG3 stimulates repression of Racl}

It's clear that lncRNAs modulate hundreds of mRNA targets, resulting in global changes in the cellular phenotype of cells. Combining with previous reports and online targeting predicting resources, we found that lncRNA MEG3 could theoretically target Rac1, a key gene regulating cell survival and metastasis. Figure 4A showed the diagram of the binding sequence of MEG3-RAC1 by NCBI blast online tools. Our following qRT-PCR and immunoblotting assay verified this prediction, which was that MEG3 overexpression induced repression of Rac1 mRNA and protein expression (Figure 4B and C). Next, we found Rac1 expression was significantly upregulated in CINs compared with NCEs and further increased in SCCs (Figure 4D), and it was negatively correlated with MEG3 level in SCC tissues (Figure 4E). Furthermore, we found that Rac1 was significantly upregulated

A
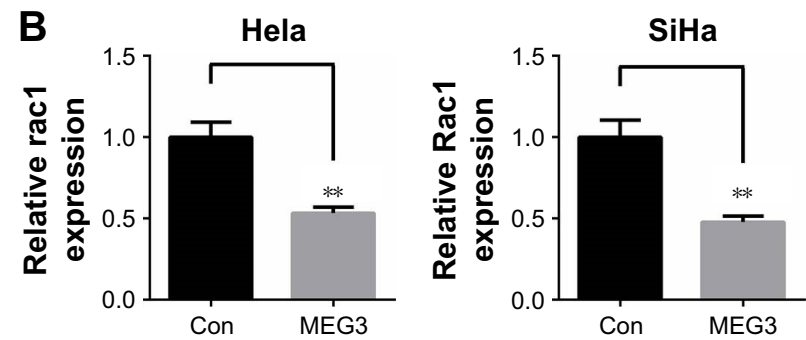

C

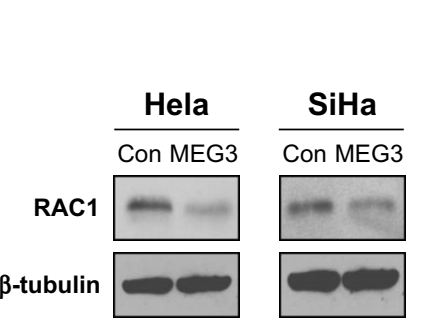

RAC1 3'-UTR 25-40 5' ... CCTGTCCCTTGGAACC ... 3' MEG3 1,526-1,541 $33^{\prime} \ldots$ GGACACGAAACCT TGG ... $5^{\prime}$
D

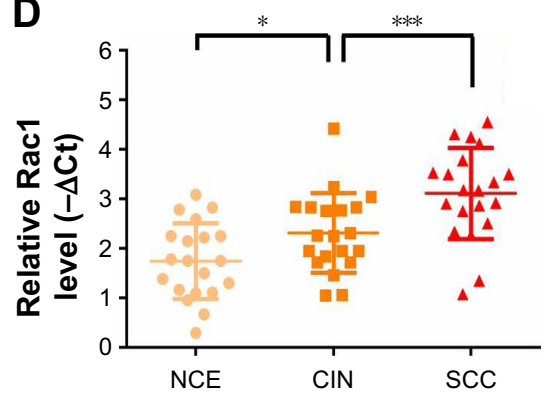

E
Linear regression

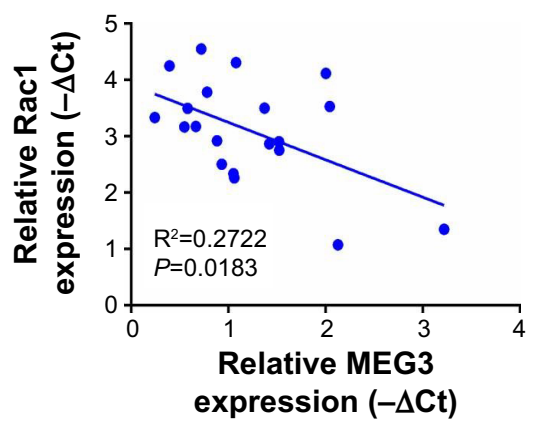

$\mathbf{F}$

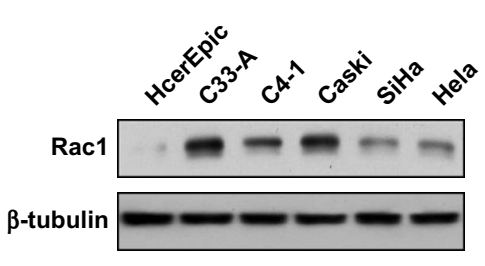

G

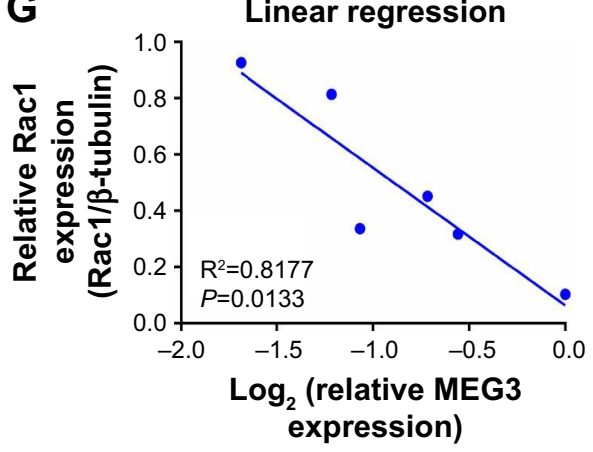

Figure 4 IncRNA MEG3 negatively regulates Racl gene expression.

Notes: (A) Diagram of the binding sequence of MEG3-RACI by blast.ncbi.nlm.nih.gov prediction. (B) MEG3 inhibited the expression of Racl at the mRNA level in both Hela and $\mathrm{SiHa}$ cells. (C) MEG3 inhibited the expression of Racl at the protein level in both Hela and SiHa cells. (D) qRT-PCR analysis of expression of IncRNA Racl expression in NCE, CIN, and SCC tissue samples $(n=20)$. $* * * P<0.001$. (E) Analysis of correlation of IncRNA MEG3 and Racl expression in SCC tissue samples ( $n=20)$ by linear regression analysis. (F) Expression of Racl protein level was detected by immunoblotting in non-tumoral HcerEpic and human cervical carcinoma cells (C-33A, C4-I, Caski, SiHa, and Hela). (G) Analysis of correlation of IncRNA MEG3 and Racl expression in non-tumoral HcerEpic and human cervical carcinoma cells (C-33A, C4-I, Caski, SiHa, and Hela) by linear regression analysis. $* P<0.05, * * P<0.01$.

Abbreviations: con, control; NCE, normal cervical epithelium; CIN, cervical intraepithelial neoplasia; SCC, squamous cell carcinoma; HcerEpic, human cervical epithelial cells; IncRNA, long non-coding RNA; MEG3, maternally expressed gene 3; qRT-PCR, quantitative RT-PCR. 
A

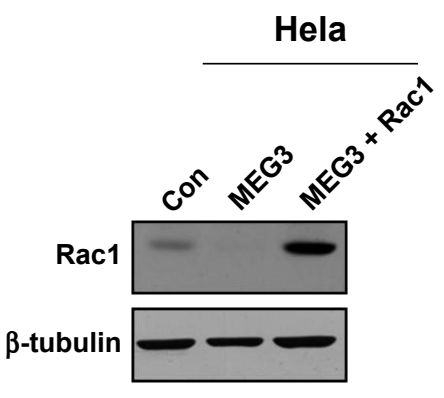

C

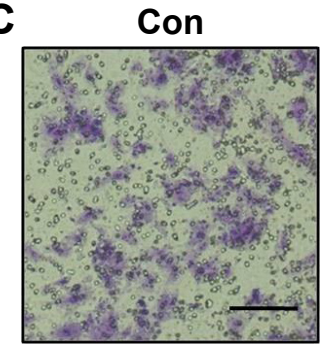

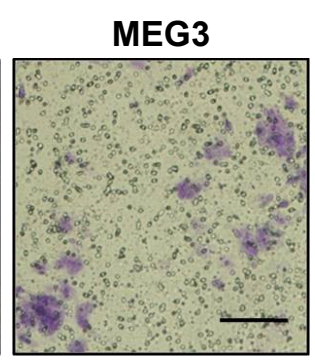

B

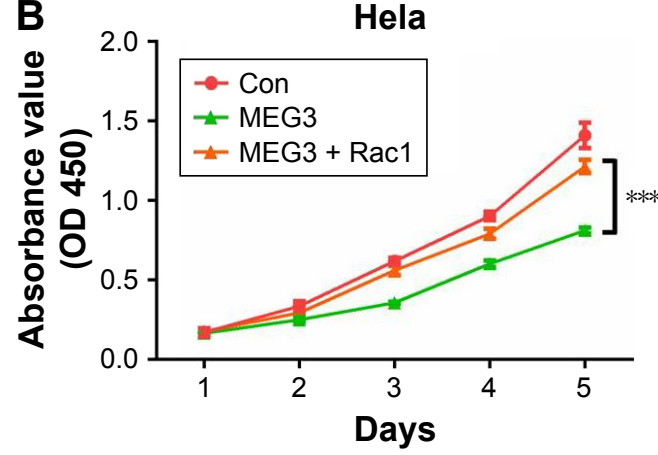

MEG3 + Rac1

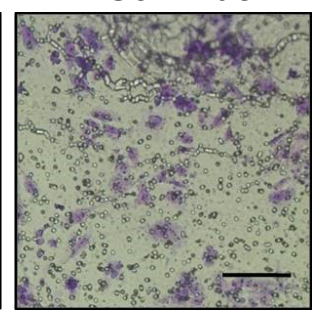

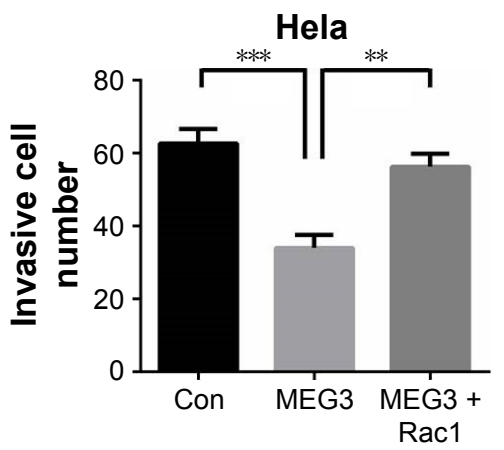

Figure 5 IncRNA MEG3 functions in cell proliferation and invasion through the Racl pathway.

Notes: (A) Immunoblotting assay was performed in Hela cells transfected with MEG3 and Racl overexpression plasmid. (B) CCK-8 assay was performed in Hela cells transfected with MEG3 and Racl overexpression plasmid. (C) Transwell assay was performed in Hela cells transfected with MEG3 and Racl overexpression plasmid. Data are presented as mean $\pm S D$. $* * P<0.01$, $* * * P<0.00$ I. Scale bar: $50 \mu \mathrm{m}$.

Abbreviations: con, control; IncRNA, long non-coding RNA; MEG3, maternally expressed gene 3.

in the abovementioned CC cell lines (Figure 4F), whose expression exhibited a clear negative linear correlation with MEG3 levels in these cell lines $(P<0.05$, Figure 4G). This finding indicated that MEG3 could exert tumor inhibitory effects on CC cells via targeting of Rac1.

\section{IncRNA MEG3 functions in cell survival and invasion through Racl pathway}

In order to evaluate whether Rac1 could reverse the inhibitory effect caused by MEG3 overexpression, rescue experiments were performed. Firstly, we overexpressed Rac1 in MEG3 overexpressing Hela cells, as shown in Figure 5A. Also, we found that Rac1 overexpression could increase the cell survival and invasion by CCK-8 and transwell assay, compared to the MEG3 overexpressing group (Figure 5B and C). This finding indicated that MEG3 did function in cell survival and invasion through the Racl pathway, at least partly.

\section{Discussion}

lncRNAs, once considered as transcription noise, now appear as vital regulators in a large range of biological processes as mentioned above..$^{9-11}$ More than 3,000 human lncRNAs have been identified, however, less than $1 \%$ of them have been characterized. ${ }^{20}$ They exert diverse actions on cancer cells through distinct mechanisms, such as imprinting, chromosome dosage-compensation, epigenetic modulation, RNA splicing, nuclear, and cytoplasmic trafficking. ${ }^{21}$ To date, many lncRNAs have been found to be involved in $\mathrm{CC}$ progression and highlighted as novel targets for diagnosis, as well as prognosis, and future targeted therapies for CC. ${ }^{22}$

In this study, we characterized that lncRNA MEG3 was downregulated in CIN and further decreased in SCC tissues, compared to NCE tissues. Although it has been confirmed by two groups, ${ }^{17,23}$ our findings further validated its expression pattern in $\mathrm{CC}$ and strongly suggested its antitumoral role in CC. In fact, we learned from lines of evidence that MEG3 expression is controlled by epigenetic regulation, that is, prevalent $\mathrm{CpG}$ methylation results in its low expression in some cancers. ${ }^{24,25}$ Also, other studies in CC have validated the promoter hypermethylation of MEG3 in both plasma and tissues of CC patients, ${ }^{26,27}$ fully supporting its downregulation in CC. On the other hand, we confirmed its low expression in several CC cell lines, compared with the non-tumoral HcerEpic cells.

In order to characterize the exact role of MEG3 in $\mathrm{CC}$, we performed overexpression assays in both $\mathrm{CC}$ cell lines, 
Hela and SiHa. As predicted, forced expressing of MEG3 suppressed cell survival as revealed by CCK- 8 assay. This was not surprising, since it has been found by Zhang's study. ${ }^{17}$ Importantly, we intriguingly found that knockdown of MEG3 promoted migration and invasion, while overexpression of MEG3 inhibited invasion of CC cells. Also, cell proliferation and EMT markers were changed consistently with the phenotype. This finding is the first time in $\mathrm{CC}$, although it has been identified in some other malignancies. ${ }^{15,28}$ Further in vivo data are needed to fully confirm this result.

Finally, we identified Rac1 as an important target of lncRNA MEG3 in CC, since MEG3 overexpression dramatically reduced its mRNA and protein levels in both Hela and SiHa cells. Wang's study in thyroid carcinoma firstly validated Rac1 as an existing target of MEG3 by specifically base pairing to its $3^{\prime}$-UTR and leading to transcription repression. ${ }^{28}$ Herein, we also support the targeting of Rac1 by MEG3. As a well-known Rho GTPase, Rac1 contributes to cancer cell survival and metastasis through various signaling pathways. And, the rescue experiments in our study confirm that MEG3 functions through Rac1 pathways. Therefore, our findings enriched the mechanism of MEG3 in determining $\mathrm{CC}$ cell behaviors, at least through targeting Rac1.

\section{Conclusion}

Our data suggest an important role of lncRNA MEG3 in the molecular biology of CC, especially in highlighting its anti-migration and anti-invasion abilities, and implicating the potential application of MEG3 in CC therapy in the future.

\section{Disclosure}

The authors report no conflicts of interest in this work.

\section{References}

1. Jemal A, Bray F, Center MM, Ferlay J, Ward E, Forman D. Global cancer statistics. CA Cancer J Clin. 2011;61(2):69-90.

2. Siegel R, Ma J, Zou Z, Jemal A. Cancer statistics, 2014. CA Cancer J Clin. 2014;64(1):9-29.

3. Galloway TJ, Ridge JA. Management of squamous cancer metastatic to cervical nodes with an unknown primary site. J Clin Oncol. 2015; 33(29):3328-3337.

4. Biglia N, Bounous VE, Sgro LG, D’Alonzo M, Gallo M. Treatment of climacteric symptoms in survivors of gynaecological cancer. Maturitas. 2015;82(3):296-298.

5. Yan J, Zhang Y, Ren C, Shi W, Chen L. Involvement of nuclear protein C23 in activation of EGFR signaling in cervical cancer. Tumour Biol. 2016;37(1):905-910.

6. Yee GP, de Souza P, Khachigian LM. Current and potential treatments for cervical cancer. Curr Cancer Drug Targets. 2013;13(2):205-220.
7. Gibb EA, Brown CJ, Lam WL. The functional role of long non-coding RNA in human carcinomas. Mol Cancer. 2011;10:38.

8. Brosnan CA, Voinnet O. The long and the short of noncoding RNAs. Curr Opin Cell Biol. 2009;21(3):416-425.

9. Huarte M. The emerging role of lncRNAs in cancer. Nat Med. 2015; 21(11):1253-1261.

10. Malek E, Jagannathan S, Driscoll JJ. Correlation of long non-coding RNA expression with metastasis, drug resistance and clinical outcome in cancer. Oncotarget. 2014;5(18):8027-8038.

11. Chen QN, Wei CC, Wang ZX, Sun M. Long non-coding RNAs in anti-cancer drug resistance. Oncotarget. 2017;8(1):1925-1936.

12. Miyoshi N, Wagatsuma $\mathrm{H}$, Wakana $\mathrm{S}$, et al. Identification of an imprinted gene, Meg3/Gt12 and its human homologue MEG3, first mapped on mouse distal chromosome 12 and human chromosome 14q. Genes Cells. 2000;5(3):211-220.

13. Zhou Y, Zhang X, Klibanski A. MEG3 noncoding RNA: a tumor suppressor. J Mol Endocrinol. 2012;48(3):R45-R53.

14. Gao Y, Lu X. Decreased expression of MEG3 contributes to retinoblastoma progression and affects retinoblastoma cell growth by regulating the activity of Wnt/ $\beta$-catenin pathway. Tumour Biol. 2016;37(2): 1461-1469.

15. Wei GH, Wang X. IncRNA MEG3 inhibit proliferation and metastasis of gastric cancer via p53 signaling pathway. Eur Rev Med Pharmacol Sci. 2017;21(17):3850-3856.

16. Qin R, Chen Z, Ding Y, Hao J, Hu J, Guo F. Long non-coding RNA MEG3 inhibits the proliferation of cervical carcinoma cells through the induction of cell cycle arrest and apoptosis. Neoplasma. 2013;60(5): 486-492.

17. Zhang J, Yao T, Wang Y, Yu J, Liu Y, Lin Z. Long noncoding RNA MEG3 is downregulated in cervical cancer and affects cell proliferation and apoptosis by regulating miR-21. Cancer Biol Ther. 2016;17(1): 104-113.

18. Kazanietz MG, Caloca MJ. The Rac GTPase in cancer: from old concepts to new paradigms. Cancer Res. 2017;77(20):5445-5451.

19. Marei H, Malliri A. Rac1 in human diseases: the therapeutic potential of targeting Rac1 signaling regulatory mechanisms. Small GTPases. 2017;8(3):139-163.

20. Kung JT, Colognori D, Lee JT. Long noncoding RNAs: past, present, and future. Genetics. 2013;193(3):651-669.

21. Zhang H, Chen Z, Wang X, Huang Z, He Z, Chen Y. Long non-coding RNA: a new player in cancer. J Hematol Oncol. 2013;6:37.

22. Peng L, Yuan X, Jiang B, Tang Z, Li GC. LncRNAs: key players and novel insights into cervical cancer. Tumour Biol. 2016;37(3):2779-2788.

23. Qin R, Chen Z, Ding Y, Hao J, Hu J, Guo F. Long non-coding RNA MEG3 inhibits the proliferation of cervical carcinoma cells through the induction of cell cycle arrest and apoptosis. Neoplasma. 2013; 60(5):486-492.

24. Li J, Bian EB, He XJ, et al. Epigenetic repression of long non-coding RNA MEG3 mediated by DNMT1 represses the p53 pathway in gliomas. Int J Oncol. 2016;48(2):723-733.

25. Zhuo H, Tang J, Lin Z, et al. The aberrant expression of MEG3 regulated by UHRF1 predicts the prognosis of hepatocellular carcinoma. Mol Carcinog. 2016;55(2):209-219.

26. Zhang J, Yao T, Lin Z, Gao Y. Aberrant methylation of MEG3 functions as a potential plasma-based biomarker for cervical cancer. Sci Rep. 2017;7(1):6271.

27. Zhang J, Lin Z, Gao Y, Yao T. Downregulation of long noncoding RNA MEG3 is associated with poor prognosis and promoter hypermethylation in cervical cancer. J Exp Clin Cancer Res. 2017;36(1):5.

28. Wang C, Yan G, Zhang Y, Jia X, Bu P. Long non-coding RNA MEG3 suppresses migration and invasion of thyroid carcinoma by targeting of Rac1. Neoplasma. 2015;62(4):541-549. 
OncoTargets and Therapy

\section{Publish your work in this journal}

OncoTargets and Therapy is an international, peer-reviewed, open access journal focusing on the pathological basis of all cancers, potential targets for therapy and treatment protocols employed to improve the management of cancer patients. The journal also focuses on the impact of management programs and new therapeutic agents and protocols on

patient perspectives such as quality of life, adherence and satisfaction. The manuscript management system is completely online and includes a very quick and fair peer-review system, which is all easy to use. Visit http://www.dovepress.com/testimonials.php to read real quotes from published authors.

Submit your manuscript here: http://www.dovepress.com/oncotargets-and-therapy-journal 